\title{
The Trend and Cause of Mortality Burden in Infancy - China, 1990-2019
}

\author{
Chenran Wang; $\mathrm{Wao} \mathrm{Xu}^{1, \#}$
}

\begin{abstract}
Introduction: China has made remarkable achievements in reducing infant deaths over the past 30 years. Meanwhile, the trend and cause composition of infant deaths have significantly changed, and the high-level years of life lost (YLLs) presents challenges to public health. This study analyzes the temporal trend and cause of infant mortality burden in China from 1990 to 2019.

Methods: Based on the updated estimates from the 2019 Global Burden of Disease Study, we used death and YLLs to describe the infant mortality burden. The percentage changes were applied to illustrate the temporal variation over the period 1990-2019.

Results: In 2019, the estimated infant deaths and YLLs in China were 101.9 (95\% uncertainty interval: 88.6-116.4) thousand and 9.0 (7.9-10.3) million, respectively, which decreased by $89.9 \%$ compared with 1990. Most infant deaths occurred in the neonatal period, especially the first 7 days of life. China's infant mortality rates for all causes decreased from 1990 to 2019, whereas certain preventable causes such as preterm birth and congenital heart anomalies accounted for increasing proportions during this period.

Conclusion and Implications for Public Health Practice: The present study reveals the major preventable causes of infant deaths in China. Decreasing neonatal mortality and improving health status of children at early stage of life have great significance on public health. This is also a solution to achieve the goal of "ending preventable child deaths" of the 2030 Sustainable Development Agenda.
\end{abstract}

\section{INTRODUCTION}

Infant mortality rate (IMR) is one of the most critical indicators of social development; the reduction of IMR is proved to be the leading contributor to prolonged life expectancy (1-2). Over the past 30 years, China has made remarkable achievements in reducing the infant mortality and achieved the Millennium Development Goals ahead of schedule. Despite the gap in IMR between China and developed countries being progressively narrowed, regional disparities between rural counties of western China and urban areas in economically developed provincial-level administrative divisions remain high (3). Furthermore, premature mortality among infants causes a high level of years of life lost (YLL) in China, which presents challenges to population health.

A nationally comprehensive quantification of trends and leading causes of infant mortality burden (described by deaths and YLLs) can help the central authority identify national priorities for improving infant health. It can also provide important insights into achieving the Sustainable Development Goals in the new era.

In this study, we conducted a nationwide analysis with the most up-to-date data from the 2019 Global Burden of Disease (GBD) Study. Our findings can improve the evidence base for drivers that might hasten the pace of progress for infant survival.

\section{METHODS}

The GBD study used dynamic epidemiological models to ensure the results were comparable globally (4). Data on death causes and mortality were derived from China Disease Surveillance Points and Death Registration, China National Maternal and Child Health Surveillance System (MCHS), and relevant epidemiological studies. Cause of Death Ensemble model as the main standardized tool was applied to estimate mortality rates. The detailed methodology has been published previously (4-5). Causes of infant death in this study were defined as per the International Statistical Classification of Diseases (10th Revision). All data obtained were nationally representative.

IMR was defined as the number of infant (aged $<1$ 
year) deaths per 1,000 live births. YLLs were calculated by multiplying deaths at each age group by global agespecific standard life expectancy. For estimated absolute number of deaths and YLLs, corresponding 95\% uncertainty intervals (UI) were calculated using the 2.5 percentile and 97.5 percentile estimates in a posterior simulation of 1,000 ordered draws, with the aim to examine uncertainty distributions deriving from random and systematic errors. This study presented the number of deaths and YLLs by age groups: early neonatal (0-6 days), late neonatal (7-28 days), and post neonatal (29-364 days). Percentage change was computed to characterize the temporal trend in mortality burden between 1990 and 2019.

Data obtained in this study were publicly available at the Institute for Health Metrics and Evaluation website and were accessed with open online tools (http://ghdx.healthdata.org/gbd-resultstool). SAS (version 9.4, SAS Institute Inc., Cary, NC, USA) and Microsoft Office Excel (version 2019, Microsoft, USA) were used to conduct all analyses.

\section{RESULTS}

Overall, the IMR of China underwent a notable decrease from 1990 to 2019, with variations in causespecific IMRs and proportions of infant deaths being observed over the period.

Table 1 shows the numbers of infant deaths in 1990, 2000, 2010, and 2019, and percentage changes over the period 1990-2019. In 2019, a total of 101.9 (95\% UI: 88.6-116.4) thousand deaths occurred in the infant period, which decreased by $89.9 \%$ from 1990 to 2019 . China's IMR decreased by $84.3 \%$ from 43.2 deaths per 1,000 live births in 1990 to 6.8 deaths per 1,000 live births in 2019, with the rate in males being consistently higher than females. The IMR in China was considerably lower than the global level (Figure 1A).

YLLs caused by infant deaths decreased considerably from 89.8 (80.4-99.3) million in 1990 to 9.0 (7.9-10.3) million in 2019. Relative changes in YLLs and deaths in infants between 1990 and 2019 were homogeneous (Table 1).

In $2019,55.4 \%$ of infant deaths occurred in the neonatal (early and late) period. The age composition of infant deaths has progressively changed during the past 30 years. In $1990,36.4 \%$ (25.5\% early-neonatal and $10.9 \%$ late-neonatal) of total infant deaths occurred during the neonatal period, and the proportion increased from $47.7 \% \quad(37.2 \%$ early-

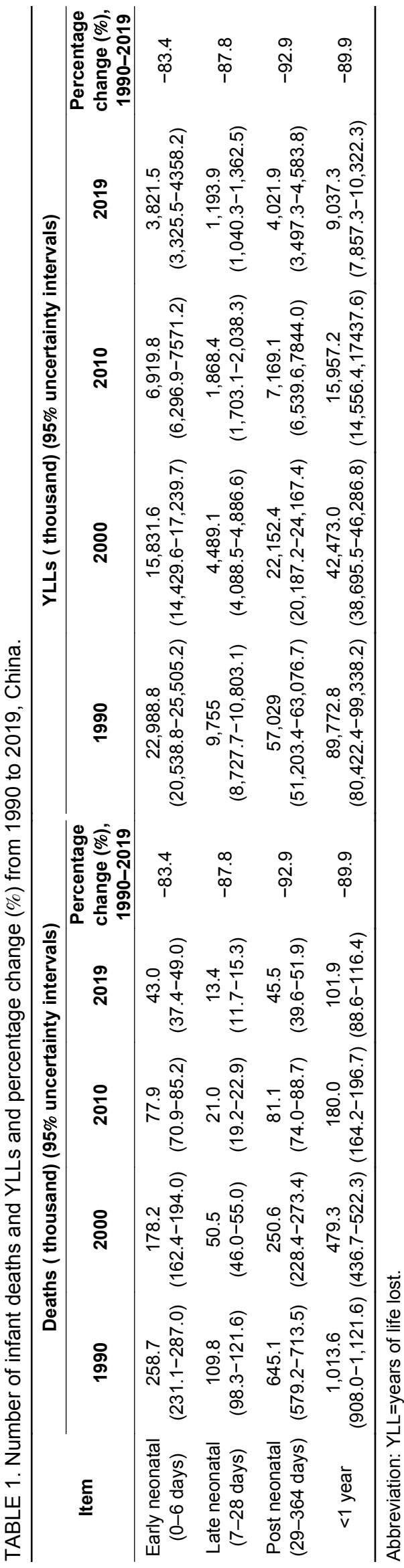


neonatal and $10.5 \%$ late neonatal) in 2000 to $54.9 \%$ (43.3\% early-neonatal and $11.6 \%$ late-neonatal) in 2010. By 2019, the age composition of infant deaths shifted to $42.2 \%$ for early-neonatal and $13.2 \%$ for lateneonatal. The trend of infant YLLs by age group was similar to that of deaths (Table 1).

Figure 1B focuses on IMRs for five main causes of infant death. In 2019, the 2 leading causes of deaths in infancy were preterm birth and congenital heart anomalies. Lower respiratory infection was the leading cause of infant death in both 1990 and 2000, whereas it fell in the fourth rank in 2019, with its IMR decreasing by $93.9 \%$ from 13.6 per 1,000 live births in 1990 to 0.8 per 1,000 live births in 2019. From 1990 to 2019 , preterm birth, congenital heart anomalies, pulmonary aspiration and foreign body in airway, and syphilis accounted for increasing proportions of deaths, whereas the proportions in neural tube defects and some infectious diseases, such as lower respiratory infection and diarrheal disease, showed an opposite

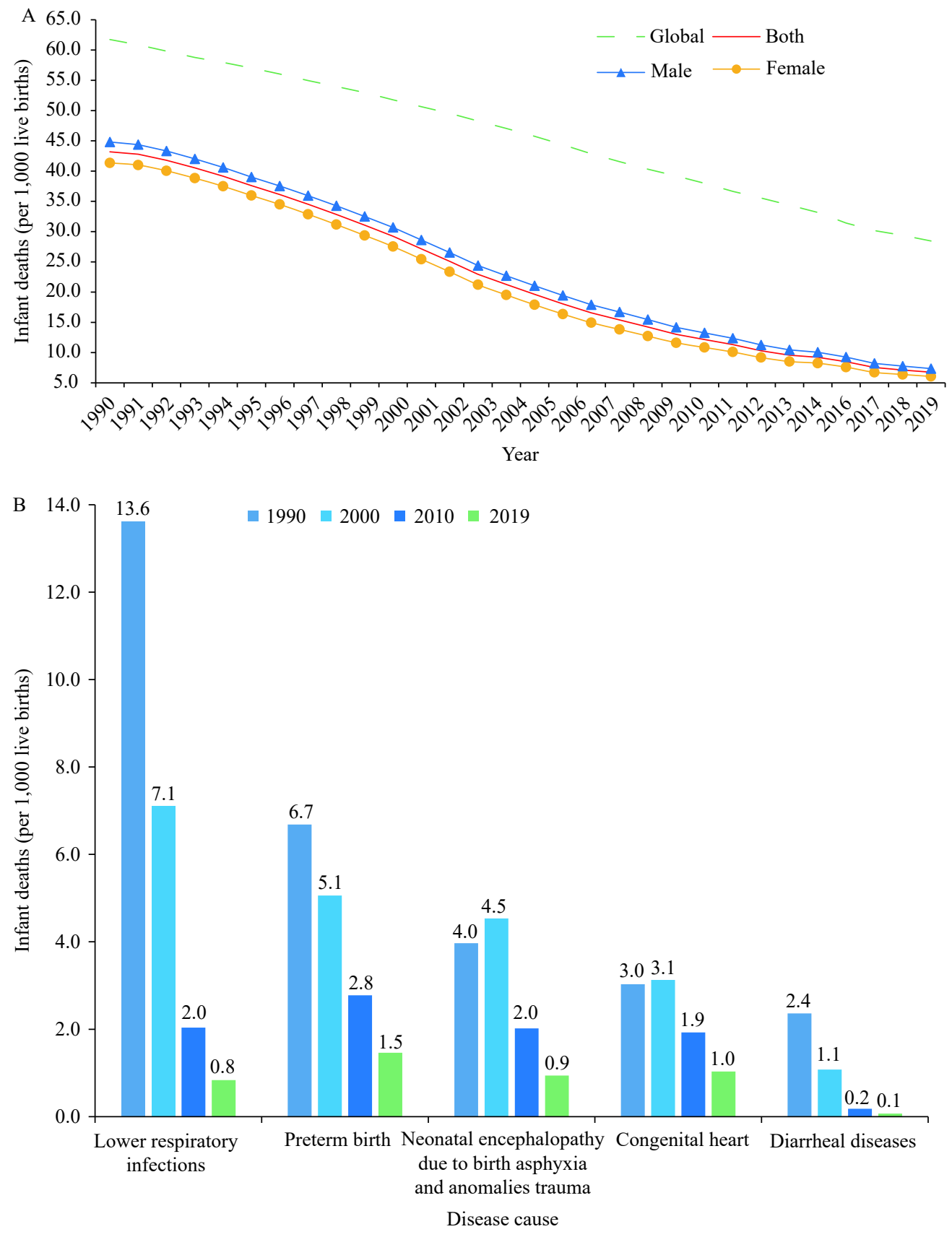

FIGURE 1. The trends of infant mortality rates by sex and cause in China, 1990-2019. (A) The trend of infant mortality rates by sex; (B) The trend of infant mortality rates by five main causes. 
downward trend. A diverging trend in proportions of deaths due to neonatal encephalopathy birth asphyxia and trauma between 1990-2000 and 2010-2019 was observed (Figure 2).

\section{DISCUSSION}

Understanding the current condition of mortality burden among infants is of vital importance to prevent health loss, which is in concordance with the target "to promote and improve women and children's health"

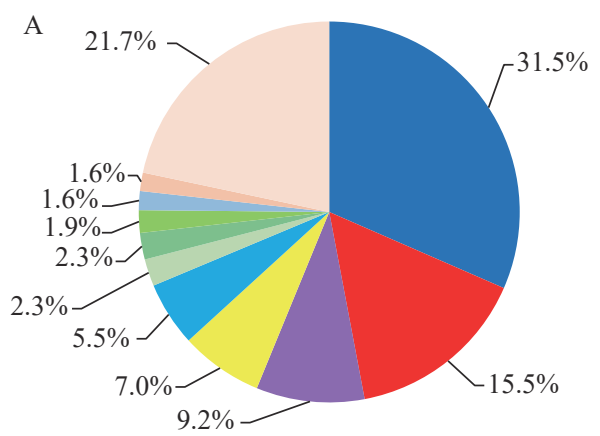

- Lower respiratory infections

- Preterm birth

- Neonatal encephalopathy due to birth asphyxia and trauma

Congenital heart anomalies

- Diarrheal diseases

- Protein-energy malnutrition

- Neural tube defects

- Pulmonary aspiration and foreign body in airway

- Meningitis

Other congenital birth defects

Other infant diseases

C

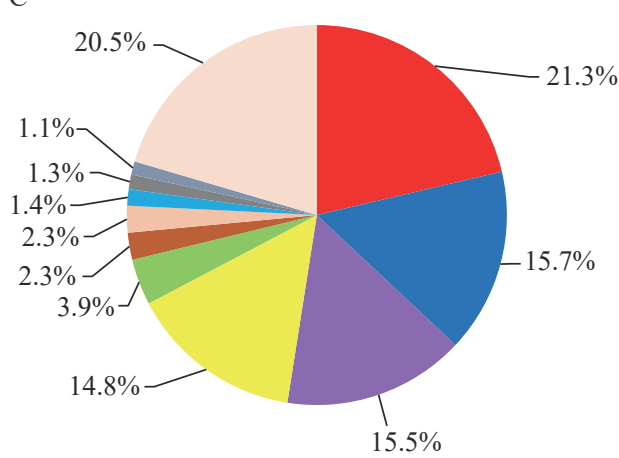

- Preterm birth

- Lower respiratory infections

- Neonatal encephalopathy due to birth asphyxia and trauma

- Congenital heart anomalies

- Pulmonary aspiration and foreign body in airway

- Digestive congenital anomalies

- Other congenital birth defects

- Diarrheal diseases

- Syphilis

- Total cancers

Other infant diseases proposed in "the $14^{\text {th }}$ Five-Year Health Development Plan" and "Healthy China 2030" (6).

Based on this study, we found that China had made tremendous progress in reducing national all-cause IMR and age-cause-specific IMRs since 1990. In 1994, the central authority introduced the Law on Maternal and Infant Health Care (7), which meant that improving maternal and infant health care as a policy priority was placed on the national development agenda. Furthermore, the implementation of health policies, as well as the improvements of income per

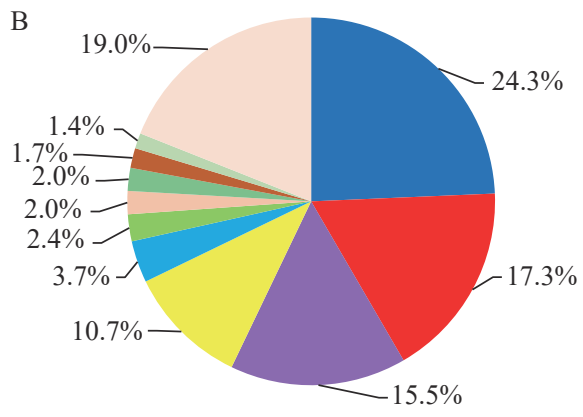

- Lower respiratory infections

- Preterm birth

- Neonatal encephalopathy due to birth asphyxia and trauma

- Congenital heart anomalies

- Diarrheal diseases

- Pulmonary aspiration and foreign body in airway

- Other congenital birth defects

- Neural tube defects

- Digestive congenital anomalies

- Protein-energy malnutrition

Other infant diseases

$\mathrm{D}$

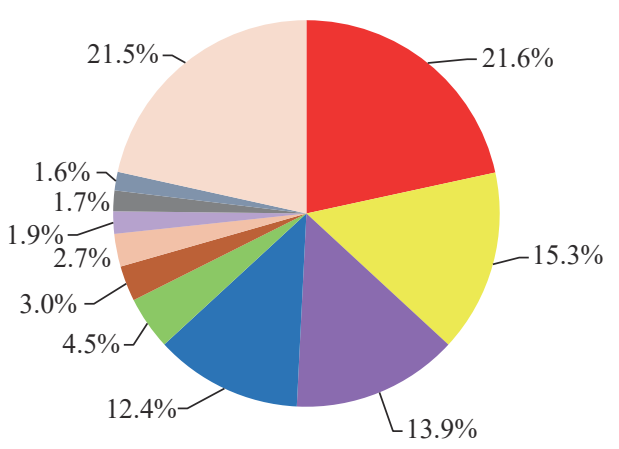

- Preterm birth

- Congenital heart anomalies

- Neonatal encephalopathy due to birth asphyxia and trauma

- Lower respiratory infections

- Pulmonary aspiration and foreign body in airway

- Digestive congenital anomalies

- Other congenital birth defects

- Neonatal sepsis and other neonatal infections

- Syphilis

- Total cancers

FIGURE 2. Proportion (\%) of causes for infant deaths from 1990 to 2019, China. (A) in 1990; (B) in 2000; (C) in 2010; (D) in 2019. 
capita, educational attainment, and medical technologies, made life-saving health interventions more available to local communities (3).

The cause composition of death has substantially changed over the past 30 years. For most causes including low respiratory infections, neural tube defects, and protein-energy malnutrition, the remarkable gains were closely related to the expansion of significant public health programs, such as "The Control and Prevention of Childhood Pneumonia," "The Expanded Program for Immunization," and "folic acid supplementation project," and the improvements of sanitation conditions, medical technologies, and maternal and child health services (1,8-9). Compared with 1990 to 2000, neonatal encephalopathy due to birth asphyxia and trauma accounted for a decreasing proportion of death from 2010 to 2019 . In order to reduce the rates of mortality and disability for neonatal asphyxia, China implemented the Neonatal Resuscitation Program since 2004 (10). Evidence showed that the implementation of neonatal resuscitation was associated with decreased incidence and mortality rates for neonatal asphyxia (11). However, the increased proportions of death attributable to preterm birth, congenital heart anomalies, pulmonary aspiration and foreign body in airway, and syphilis underscore the needs of strengthening antenatal care, monitoring congenital anomalies, preventing unintentional infant injury, and intensifying the National Prevention of Mother-to-Child Transmission programme in future work (9).

By examining the age composition of infant mortality, we found that most infant deaths occurred in the neonatal period, especially the first seven days of life. In order to mitigate the mortality burden efficiently, interventions targeted to reduce preventable neonatal death should be implemented. The World Health Organization recommended intervention, Early Essential Newborn Care that contains a package of interventions to reduce neonatal mortality during childbirth and within the first few days after delivery, was such an attempt (12-13). National scale-up of similar practices is recommended to further promote infants' health.

This study was subject to some limitations. First, as we conducted this study at the national level, the gap in IMR between the national and sub-national levels underscores the need to undertake more specific assessments at the province/county and urban/rural levels in future studies. Second, with regard to the estimation of infant mortality, GBD used a unified methodology to ensure the results were comparable globally, which means data on mortality in this study may be slightly different from other data source such as MCHS. Nevertheless, this is the first study that provides the most up-to-date and comprehensive estimates of infants' mortality burden in China from 1990 to 2019. Our findings provide useful evidence for tailored strategies that consider highly vulnerable neonates and major causes of infant deaths and are expected to be adopted by the central and provincial health authorities.

Conflicts of interest: No conflicts of interest were reported.

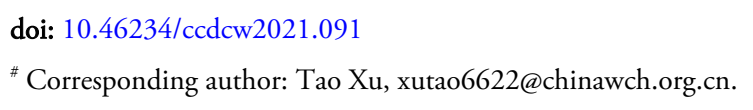

${ }^{1}$ National Center for Women and Children's Health, Chinese Center for Disease Control and Prevention, Beijing, China.

Submitted: January 12, 2021; Accepted: April 13, 2021

\section{REFERENCES}

1. GBD 2015 Child Mortality Collaborators. Global, regional, national, and selected subnational levels of stillbirths, neonatal, infant, and under-5 mortality, 1980-2015: a systematic analysis for the Global Burden of Disease Study 2015. Lancet 2016;388(10053):1725- 74 . http://dx.doi.org/10.1016/S0140-6736(16)31575-6.

2. Xu YH, Zhang WF, Yang RL, Zou CC, Zhao ZY. Infant mortality and life expectancy in China. Med Sci Monit 2014;20:379-85. http://dx.doi.org/10.12659/MSM.890204.

3. Wang YP, Li XH, Zhou MG, Luo SS, Liang J, Liddell CA, et al. Under-5 mortality in 2851 Chinese counties, 1996-2012: a subnational assessment of achieving MDG 4 goals in China. Lancet 2016; 387(10015):273 - 83. http://dx.doi.org/10.1016/S0140-6736(15)00 554-1.

4. GBD 2019 Diseases and Injuries Collaborators. Global burden of 369 diseases and injuries in 204 countries and territories, 1990-2019: a systematic analysis for the Global Burden of Disease Study 2019. Lancet 2020;396(10258):1204 - 22. http://dx.doi.org/10.1016/S0140-6736 (20)30925-9.

5. GBD 2017 Disease and Injury Incidence and Prevalence Collaborators. Global, regional, and national incidence, prevalence, and years lived with disability for 354 diseases and injuries for 195 countries and territories, 1990-2017: a systematic analysis for the Global Burden of Disease Study 2017. Lancet 2018;392(10159):1789 - 858. http://dx. doi.org/10.1016/S0140-6736(18)32279-7.

6. Tan XD, Zhang YN, Shao HY. Healthy China 2030, a breakthrough for improving health. Glob Health Promot 2019;26(4):96-9. http://dx.doi.org/10.1177/1757975917743533.

7. The Central People's Government of the People's Republic of China. Maternal and Child Health Law of the People's Republic of China. http://www.gov.cn/banshi/2005-08/01/content_18943.htm. [2021-0415]. (In Chinese).

8. MoH. Management plan of folic acid supplementation to prevent neural tube defects programme. Beijing, 2009. http://www.nhc.gov. cn/fys/s3581/201006/942109bebb4340b2922898f565489a6f.shtml. [2021-04-10]. (In Chinese).

9. Zhang T. Health care for women and children in China: over half the sky (Public health in China Series). Beijing: People's medical publishing 
house, 2019: 100-72. (In Chinese)

10. Xu T, Wang HS, Gong LM, Ye HM, Yu RJ, Wang DH, et al. The impact of an intervention package promoting effective neonatal resuscitation training in rural China. Resuscitation 2014;85(2):253 - 9. http://dx.doi.org/10.1016/j.resuscitation.2013.10.020.

11. Xu T, Yue Q, Wang HS, Ye HM, Yu RJ, Wang DH, et al. Evaluation on the second phase of Neonatal Resuscitation Program in China. Chin J Perinat Med 2017;20(5):346 - 51. http://dx.doi.org/10.3760/cma.j. issn.1007-9408.2017.05.009. (In Chinese).
12. Xu T, Qu W, Wang Y, Yue Q, Huang XN, Tian XB. Preplanned studies: analysis of early essential newborn care capacities of rural health facilities-four provinces in Western China, 2016. China CDC Wkly 2020;2(1):8 - 12. http://dx.doi.org/10.46234/ccdcw2020.002.

13. Qu W, Yue Q, Wang Y, Yang JL, Jin X, Huang XN, et al. Assessing the changes in childbirth care practices and neonatal outcomes in Western China: pre-comparison and post-comparison study on early essential newborn care interventions. BMJ Open 2020;10(12):e041829. http://dx.doi.org/10.1136/bmjopen-2020-041829. 